\title{
Performance Monitoring using Regression Approach in ATM Networks*
}

\author{
Hon-Wai Chu ${ }^{\mathrm{a}}$ and Danny H. K. Tsang ${ }^{\mathrm{b}}$

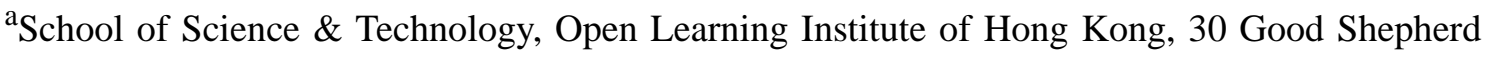 \\ Street., Kowloon, Hong Kong. \\ ${ }^{\mathrm{b}}$ Department of Electrical \& Electronic Engineering, The Hong Kong University of Science \& \\ Technology, Clear Water Bay, Kowloon, Hong Kong
}

\begin{abstract}
A new performance monitoring mechanism is proposed to monitor stringent cell loss ratio (CLR), e.g. $10^{-9}$, within a reasonable time window. The basic idea is to use several virtual buffers whose buffer sizes or service capacities are much smaller than that of the physical buffer. The resulting higher CLR in the virtual buffers can be monitored accurately in small measurement periods. These monitored values at virtual buffers are combined with a model in a regression algorithm to estimate the actual CLR of the physical system. This approach has several advantages: 1) It can shorten the measurement period to estimate small CLR. 2) We can use existing performance estimation approaches as our model and the new estimate is simply obtained by regression. 3) This approach works even if we have only a few measured data. 4) It is not necessary to know the behaviour of the actual system because we infer information directly from measurement.
\end{abstract}

\section{INTRODUCTION}

ATM takes advantage of statistical multiplexing of bursty sources to allow efficient sharing of communication resources among users. The service provider has to ensure that there are sufficient resources to meet the user's specified quality of service (QoS). This normally involves two procedures: 1) performance estimation and 2) performance monitoring. The network first uses performance estimation methods to estimate the resource required for a new call. Based on this estimation, the network decides the call admission and reserves resources when the call is accepted. Afterwards, the network continues to monitor the traffic to confirm that the actual QoS meets the target QoS. If a QoS violation is detected, the network has to adjust the resource allocation. The performance estimation and performance monitoring are essential for the network management, congestion control and resources dimensioning.

Most of the previous studies concentrate on performance estimation method and various approaches have been proposed [1][7][8][9]. On the contrary, very few works have been dedicated to the field of performance monitoring. The major difficulty of performance monitoring is the real-time requirement. In other words, the monitoring mechanism must be able to detect a QoS violation as fast as possible. In this paper, the QoS parameters that we are mostly interested in is cell loss ratio (CLR).

Conceptually, CLR can be estimated by counting the fraction of cell loss over a fixed period of time in an ATM switch. It can be implemented easily with simple counters if the target

* Supported in part by Hongkong Telecom Institute of Information Technology grant HKTIIT93/

94.EG01 and in part by RGC-competitive Earmarked Research Grant HKUST 780/96E. 
CLR can be measured accurately within in a reasonable time interval, e.g. CLR $\geq 10^{-3}$. Nevertheless, stringent CLR requirement is often specified in the range of $10^{-9}$. This makes monitoring by direct statistical methods impossible for such small CLR. For example, we need at least $10^{11}$ cell arrivals to detect a CLR of $10^{-9}$ with acceptable confidence. Assuming a link rate of $155 \mathrm{Mb} / \mathrm{s}$ and a load of $90 \%$ on the link, this would require about 3.5 days. Due to call arrivals or departures, the traffic conditions may have already changed well before any meaningful statistics are collected. Therefore, we need some other methods to shorten the measurement periods.

In this paper, we propose a new performance monitoring mechanism to monitor stringent CLR, e.g. $10^{-9}$, within a reasonable time window. The basic idea is to use several virtual buffers whose buffer sizes or service capacities are much smaller than that of the physical buffer. Thus, we can increase the cell losses observed in the virtual buffers. These monitored values at virtual buffers are combined with a model in a regression algorithm to estimate the actual CLR of the physical system. This approach can detect QoS violation even there is no cell loss observed in the physical system. Accordingly, corrective actions can be activated to prevent possible future cell losses.

Traffic monitoring mechanisms based on model and measurement are also proposed in [3] and [15]. The work in [15] is different from us in that it first selects the asymptotic relationship between buffer and CLR using hypothesis testing. Based on this asymptotic relationship, a simple regression model is established to predict small CLR. We shall show that this is a special case of our work in which a specific model based on asymptotic behaviour of buffer is used. While in [3] a better asymptotic relationship is proposed. Instead of using the asymptotic behaviour model, we can apply other models in our proposed regression. This gives us more flexibility because our regression approach does not depend only on asymptotic model. Furthermore, our regression approach can also be applied to service capacity instead of buffer.

In [14], Saito has considered a similar problem using Bayesian analysis. Nevertheless, there is no clear explanation of the choice of the function being used in his works. Furthermore, the choices of certain parameters are not clear there and hence the actual implementation of the algorithm may cause problems. In our approach, we use well-defined parameters for our regression function.

The paper is organized as follows. Section 2 introduces the virtual output buffer mechanism to monitor the QoS. Section 3 reviews the basic idea of regression approach. Section 4 evaluates the performance of regression with numerical examples. Section 5 presents our conclusions.

\section{VIRTUAL OUTPUT BUFFER MECHANISM}

The aim of our monitoring mechanism is to estimate the actual CLR in the range of

$10^{-9}$, which cannot be measured by direct observation. It is well known that cell loss increases monotonically with decreasing buffer size or service capacity. Hence, if we reduce the buffer size and/or service capacity, more cell losses will be observed. For example, CLR in the range of $10^{-2}$ to $10^{-3}$ can be estimated accurately with only $10^{5}$ cell arrivals, which is feasible in a short period.

The basic idea is to use several virtual buffers whose buffer size or service capacity is much smaller than the original value. We shall denote this approach as the Virtual Output Buffer (VOB) approach because virtual buffers are used. Figure 1 shows a system model of VOB method using $N$ virtual buffers. Let $K$ and $C$ be the actual buffer size and service capacity of the physical system, and let $K_{n}$ and $C_{n}$ be the buffer size and service capacity of the $n^{\text {th }}$ virtual buffer respectively, $n=1, \ldots, N$. The VOB method can be implemented in two different ways: 1) 


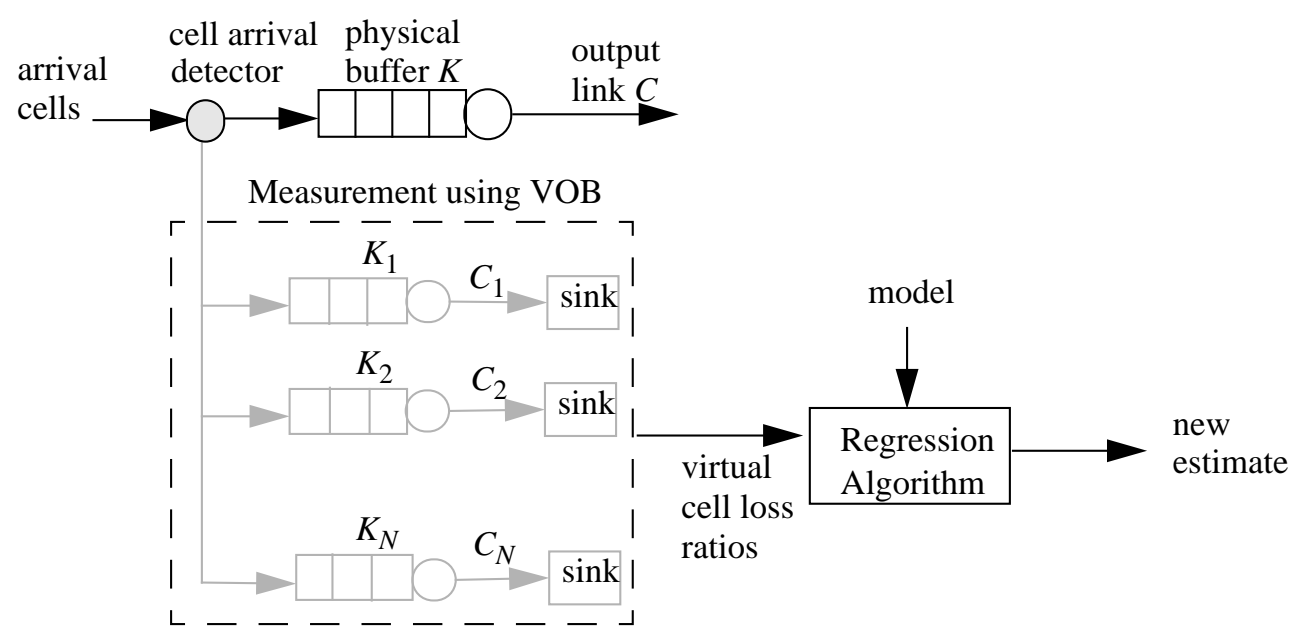

Figure 1. Virtual output buffer mechanism

Reduced buffer - the virtual buffer sizes are much smaller than the physical buffer but their service capacities are the same as the physical system, (i.e. $K_{1}<K_{2}<\ldots<K_{N}<K$ and $C_{1}=C_{2}$ $\left.=\ldots=C_{N}=C\right)$. 2) Reduced capacity - the service capacities for the virtual buffers are smaller than that of the physical system but their buffer sizes are the same as the physical buffer, (i.e. $K_{1}$ $=K_{2}=\ldots=K_{N}=K$ and $\left.C_{1}<C_{2}<\ldots<C_{N}<C\right)$. The option of reducing both buffer size and service capacity is not considered in this work. Similar ideas using virtual buffers are also proposed in the literatures. Reduced buffer approach is adopted in [3][4][15] while reduced capacity approach is used in [11] to train a neural network.

Assume the arrival process during the short measurement period is stationary. When a cell arrives at the physical buffer, it triggers a virtual cell to arrive at each virtual buffer (i.e. the counter is incremented by one). The $n^{\text {th }}$ virtual buffer is decremented at constant rate according to its service rate $C_{n}$. If the reduced buffer method is applied, each virtual buffer will be served with the same allocated bandwidth as the physical buffer. The buffer occupancy of each virtual buffer is monitored. For a given total cell arrivals $\mathcal{A}$, say $10^{5}$, the number of lost virtual cells $\mathcal{L}(n)$ in the $n^{\text {th }}$ virtual buffer are counted. Then the virtual cell loss ratio $P(n)$ of the $n^{\text {th }}$ virtual buffer is simply equal to $\mathcal{L}(n) / \mathcal{A}$. Without loss of generality, we assume that the virtual buffer sizes of $N$ buffers are $K_{1}<K_{2}<\ldots<K_{N}$. Then we have $P(1)>P(2)>\ldots>P(N)>$ CLR of the physical buffer. Similarly, we have service rates $C_{1}<C_{2}<\ldots<C_{N}<C$ for $N$ virtual buffers using the reduced capacity method. Again, we have $P(1)>P(2)>\ldots>P(N)>$ CLR of the physical buffer.

The problem is how to relate the virtual CLR $P(n)$ to the actual CLR of the physical buffer. We propose to use a regression approach to extrapolate the curve of CLR vs buffer size or CLR vs capacity. Based on the extrapolation, we can estimate the CLR of the physical buffer.

\section{REGRESSION APPROACH}

In this section, we introduce the basic idea of our regression method. We first take a model to generate the initial estimate of the QoS of the physical buffer. Due to modeling error or errors in declared parameters, this initial estimate may be very different from the actual QoS. To improve the estimate, we measure the actual QoS of the system under certain system parameters (i.e., reduced buffer sizes or reduced capacities) using the VOB method in Section 3.2. The monitored data and the initial estimate are then used in a regression algorithm to infer a new QoS estimate. This new estimate is known as regressed estimate.

Let $a(t)$ be the actual QoS of the system under system parameter $t$, which can be either 
buffer size or service capacity. Let $m(t)$ be the QoS predicted by a model. We would like to construct a function to relate $a(t)$ and $m(t)$. The exact relationship between the actual QoS $a(t)$ and the QoS for model $m(t)$ is unknown; otherwise we can directly construct $a(t)$ from $m(t)$ based on the relationship. It is always desirable to start with a simple function and to improve it later. Therefore, the following simple function is assumed:

$a(t)=c(1)+c(2) t+c(3) m(t)$

where $c(j)$ 's, $j=1,2,3$, are constant coefficients to be determined.

Suppose we measure the actual QoS $a\left(t_{i}\right)$ under system parameter $t_{i}, i=1, \ldots, N$. As in other studies [14][15], we assume that the QoS can be measured accurately. This is a reasonable assumption if the monitored CLR is large, such as $10^{-2}$ to $10^{-3}$. Then the coefficients $c(j)$ can be determined by minimizing the least squares error as follow:

$\min \sum_{i=1}^{N}\left[a\left(t_{i}\right)-\left(c(1)+c(2) t_{i}+c(3) m\left(t_{i}\right)\right)\right]^{2}$

The $c(j)$ 's are determined by the solution to this system of equations:

$$
\left\{\begin{array}{l}
N c(1)+c(2) \sum_{i=1}^{N} t_{i}+c(3) \sum_{i=1}^{N} m\left(t_{i}\right)=\sum_{i=1}^{N} a\left(t_{i}\right) \\
c(1) \sum_{i=1}^{N} t_{i}+c(2) \sum_{i=1}^{N} t_{i}^{2}+c(3) \sum_{i=1}^{N} m\left(t_{i}\right) t_{i}=\sum_{i=1}^{N} a\left(t_{i}\right) t_{i} \\
c(1) \sum_{i=1}^{N} m\left(t_{i}\right)+c(2) \sum_{i=1}^{N} m\left(t_{i}\right) t_{i}+c(3) \sum_{i=1}^{N} m^{2}\left(t_{i}\right)=\sum_{i=1}^{N} a\left(t_{i}\right) m\left(t_{i}\right)
\end{array}\right.
$$

Eq. (1) is a linear function to relate $a(t)$ and $m(t)$. We find that (1) is an appropriate function to be used in ATM problems and there are good reasons to support this claim, as explained in the following subsections.

\subsection{Reduced Buffer Approach}

If the reduced buffer approach is used, the system parameter $t$ will become buffer size. It is well known that [13] there are two scales of congestion in an ATM multiplexer: 1) cell scale congestion for small buffer and 2) burst scale congestion for large buffer. Note that the buffer size is measured relative to the length of a burst. That is, large buffer means that the buffer size is of several burst lengths, while small buffer generally means that the buffer size is much smaller than one burst length. Particularly for large buffer, there exists an asymptotic linear relationship between logarithm of buffer overflow probability and buffer size [1][7] for models that do not possess long-range dependence. That is,

$P(x>t) \sim \gamma \exp (-\delta t) \quad \Rightarrow \log (P(x>t)) \sim \log (\gamma)-\delta t \quad$ for $t \rightarrow \infty$

where $\gamma$ and $\delta$ are both positive constants, and $x$ is the buffer occupancy.

Eq. (4) is known as asymptotic approximation in [1] and has been the focus of many studies in the literatures. Particularly, Elwalid [7] proposed the following approximation which can be used to estimate CLR for general Markovian sources:

$\log (C L R) \sim-\gamma_{0}-\delta B$

where $B$ is the buffer size, and $\gamma_{0}$ and $\delta$ are positive constants.

Suppose we use Eq. (5) as our model $m(B)$ to obtain an initial estimate of $\log (C L R)$, i.e. $m(B)=-\gamma_{0}-\delta B$. Then the regressed estimate $\hat{a}(B)$ using (1) is given by:

$$
\begin{aligned}
\hat{a}(B) & =c(1)+c(2) B+c(3)\left(-\gamma_{0}-\delta B\right) \\
& =\hat{c}(1)+\hat{c}(2) B
\end{aligned}
$$


where $\hat{c}(1)=\left(c(1)-\gamma_{0} c(3)\right)$ and $\hat{c}(2)=(c(2)-\delta c(3))$.

Thus, our regression method with the choice of (5) as the model for the initial estimate leads to a linear relationship between $\log (C L R)$ and buffer size $B$. The coefficients $\hat{c}(1)$ and $\hat{c}(2)$ are determined by measurement. As a matter of fact, Eq. (6) is the regression model being used in [15] and it is a special case of (1). Therefore, (1) is an appropriate choice for reduced buffer method.

One problem of (6) is that it assumes an explicit linear relationship between the actual QoS $a(B)$ and buffer size $B$, while no such assumption is made in (1). Instead, we assume a linear relationship between $a(B)$ and $m(B)$ but do not specify the relationship between $m(B)$ and $B$. In fact, $m(B)$ can be found by numerical methods or even simulation. Therefore, the relationship between $a(B)$ and $B$ given by (1) can be non-linear and this gives us more flexibility than using (6). Furthermore, (6) is an asymptotic approximation and is accurate only for large buffer. If we monitor the CLR using virtual buffers with small buffer sizes, (6) does not necessary to hold. In fact, if the sizes of the virtual buffers are too small, the virtual buffers may have operated in the cell scale congestion [13]. Therefore, the estimate based on (6) can be very poor and we shall illustrate this problem in our numerical examples in Section 3.5.

Recently, [8] [12] show that real traffic traces are self-similar. The long-range dependence behaviour in these traces cannot be characterized by conventional Markovian traffic model and thus the asymptotic relationship in (5) is incorrect. In [5], the following new relation is proposed:

$\log (C L R) \sim-\delta B^{2(1-H)}, \quad$ for some $H>0.5$,

where $H$ is known as the Hurst parameter and $\delta$ is a positive constant.

Nevertheless, Eq. (7) is only a rough approximation and seems to hold only for very large buffer. Further investigation is required before (7) can be used in reliable performance estimation. Thus we shall not use (7) in our work. In addition, the CLR for self-similar traffic decreases very slowly with increasing buffer size [8][12]. This small difference in CLRs shows that the reduced buffer method does not help to shorten the estimation time of small CLR values for self-similar traffic. Hence we shall use the reduced capacity approach instead.

\subsection{Reduced Capacity Approach}

There are very few works [6][9] dedicated to find relationship between CLR and service capacity in the literatures. The simplest relationship is the stationary Gaussian approximation [9], which is reasonably accurate for a large number of aggregate sources. Let $\mu_{i}$ and $\sigma_{i}$ be the mean and standard deviation of the cell arrival rate of the $i^{\text {th }}$ source. Suppose the aggregate cell arrival rate distribution is Gaussian. The required bandwidth $C$ to guarantee a CLR $\varepsilon$ for $N$ multiplexed sources is given by

$$
C=\mu+\sigma \sqrt{(-2 \log \varepsilon-\log (2 \pi))} \quad \Rightarrow \log \varepsilon=-\frac{1}{2}\left(\left(\frac{C-\mu}{\sigma}\right)^{2}+\log (2 \pi)\right),
$$

where $\mu=\sum_{i=1}^{N} \mu_{i}$ and $\sigma^{2}=\sum_{i=1}^{N} \sigma_{i}^{2}$.

It is clear from (8) that a quadratic relationship between $\log (C L R)$ and service capacity exists. If we use the Gaussian approximation as the model $m(C)$ to give the initial estimate, the linear regression in (1) will generate another quadratic relationship. That is,

$\log (C L R)=\beta_{0} C^{2}+\beta_{1} C+\beta_{2}$,

where $\beta_{0}, \beta_{1}$ and $\beta_{2}$ are constants determined by measurement. Thus, (1) is also a reasonable choice for reduced capacity approach. 


\section{NUMERICAL STUDIES AND DISCUSSIONS}

In this section, we investigate the performance of the different regression methods in predicting the actual QoS. We first start with the reduced buffer approach for Markovian sources and show the accuracy of the regressed estimates. Then we show an example of reduced capacity approach for self-similar video trace. We shall show that Eq. (1) can give accurate regressed estimates, especially in the case that there exists a linear relationship between $a(t)$ and $m(t)$.

\subsection{Reduced Buffer}

We start with a very popular model used in the literatures: Stochastic Fluid Flow model (SFF) [1]. In the simplest case, we assume that each source is operating in two states: on and off. The source is transmitting at a constant rate $R$ in the on state, while there is no transmission in the off state. We can base on this model to do queueing analysis to estimate buffer overflow probability, delay etc. The stationary buffer overflow probability $P\{x>t\}$ is given by:

$$
P\{x>t\}=\sum_{i} a_{i} e^{z_{i} t}
$$

where $x$ is buffer occupancy, $a_{i}$ are coefficients determined from the boundary conditions [1] and $z_{i}$ are the eigenvalues with negative real part.

Most of the SFF approaches assume that the on and off periods are both exponentially distributed, which may not be true in general. Recently, real traffic traces [8][12], such as Ethernet and video, have shown to exhibit self-similar behaviour. This further disproves the assumption of exponential distribution. Although there are some works to extend SFF to more general distributions [2], there are still problems in applying it to practical cases. It is because we must know the distribution of the sources in advance. If we just apply SFF without knowing the exact distribution of the sources, the estimated performance will be very poor.

\subsubsection{Example 1}

Let us consider a simple example to illustrate the problem of incorrect distribution assumption. We consider traffic source "Data" with traffic characteristics shown in Table 1.

Table 1: Traffic characteristics of on-off sources

\begin{tabular}{lccc}
\hline Source & Peak rate $(\mathrm{Mb} / \mathrm{s})$ & Mean rate $(\mathrm{Mb} / \mathrm{s})$ & Mean burst length (cells) \\
\hline Data & 10 & 1 & 339 \\
\hline
\end{tabular}

Suppose there are 20 such sources multiplexed into a finite size buffer with fixed service capacity $50 \mathrm{Mb} / \mathrm{s}$. In practice, sources can only specify their traffic characteristics in terms of parameters like those in Table 1 but not their distributions. It is because the sources may not even know the distributions. To use SFF model in (10), we assume that the on-off periods for the sources are exponentially distributed. Nevertheless, the real distributions of both on and off periods are taken to be deterministic instead. Therefore, we would like to compare the errors due to the wrong distribution assumption. In Figure 2, we first obtain the buffer overflow probability of the deterministic on-off sources by simulation. The buffer overflow probability is simulated long enough so that it is accurate up to $10^{-5}$. Then those points smaller than $10^{-5}$ are obtained by extrapolation. We compare the simulation results with those predicted by the SFF model (10). Figure 2 shows that SFF over-estimate the buffer overflow probability by orders of magnitude (e.g., $10^{-5}$ vs $10^{-9}$ at buffer size of 6 burst lengths). Consequently, the assumption of exponential distribution can be very harmful to performance estimation in terms of under-utilizing network resources in this case. 


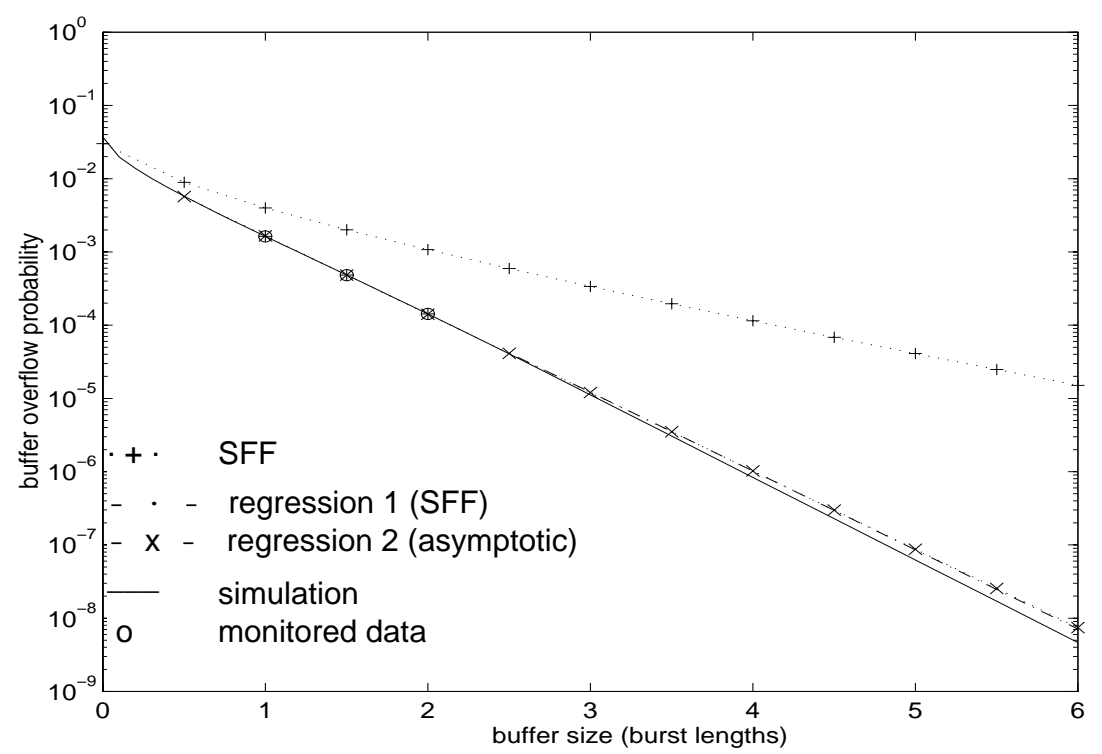

Figure 2. Comparison of simulation, model predicted and regressed results.

Instead of using more complicated models [2] to improve the estimate, we would like to apply the regression method discussed in Section 3. Here, the system parameter $t$ is buffer size (in burst length) and the QoS of interest is buffer overflow probability $P(x>t)$, where $x$ is buffer occupancy. To handle the wide range of $P(x>t)$ (e.g., 1 to $10^{-9}$ ), we use logarithm of $P(x>t)$ for both $a(t)$ and $m(t)$ in (1). Moreover, this helps to reduce the numerical errors in computations.

Suppose we monitor the buffer overflow probabilities of three virtual buffers with size of $t=1,1.5$ and 2 burst lengths respectively, and the monitored data are shown as circles in Figure 2. We first take SFF in (10) as the model for $m(t)$ to perform the regression using (1). The regressed estimate $\hat{a}(t)$ called "regression 1" is shown in Figure 2 and it is much better than using the original SFF model. We then repeat the regression using the asymptotic result in (6) to produce another set of estimates called "regression 2". Figure 2 shows that "regression 2" also gives a close estimate to the exact curve. In summary, both regression generate close estimates to the correct values. For instance, the regressed estimates $\hat{a}(t)$ are about twice the value of $a(t)$ at buffer size of 6 burst length.

This close prediction is expected because of the traffic model used. As discussed in Section 3.3, there exists a linear relationship between logarithm of buffer overflow probability and buffer size [1][7] for models which do not posses long-range dependence. Figure 2 shows that (4) holds for both the SFF model and the deterministic on-off sources, i.e. both curves $a(t)$ and $m(t)$ appear as straight lines at large buffer size. In fact, the deterministic sources exhibit this linear behaviour (in log scale) even for small buffer (e.g. burst length $=1$ ), while the SFF model exhibit the linear behaviour (in log scale) only for large buffer (burst length $>3$ ). Eq. (1) is essentially fitting a linear function between two straight lines (simulation and the SFF model in the asymptotic region), which is expected to work. Hence, simple regression like (1) can give accurate performance estimation with a few monitored data. In performance monitoring, we normally require the accuracy of CLR up to the order of magnitude. Thus, the regressed estimate is sufficient for this case. 


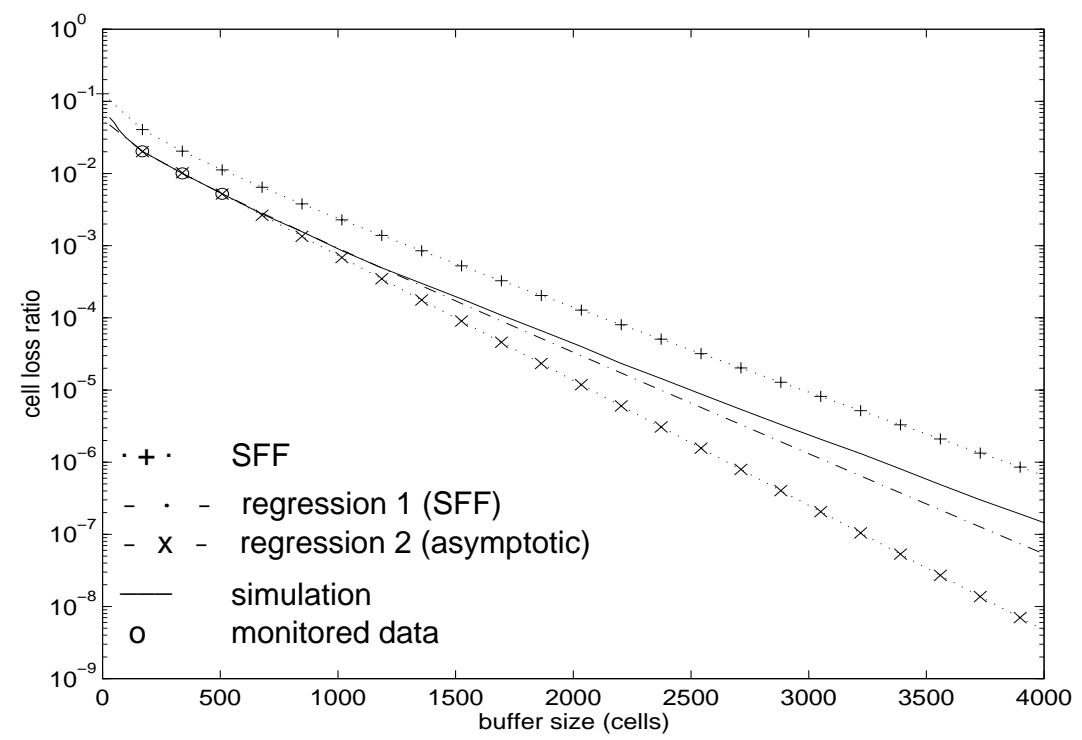

Figure 3. Regression using virtual buffers with size $=170$ cells, 340 cells and 510 cells.

\subsubsection{Example 2}

In this example, we show that if the buffer sizes of the virtual buffer becomes small, the regression based on asymptotic model (6) can generate very poor estimates. Similar to the last example, we consider traffic source "Data" with traffic characteristics shown in Table 1. Suppose there are 10 such sources multiplexed into a finite size buffer with fixed service capacity $25 \mathrm{Mb} / \mathrm{s}$. Here, the on-off periods for the sources are taken to be exponentially distributed, which agree with the assumption made in the SFF model. Therefore, the SFF model is expected to produce a close estimate of exact curve, which is shown in Figure 3. Note that the SFF overestimates the exact CLR because buffer overflow probability instead of CLR is given by SFF.

Suppose we monitor the cell loss ratios of three virtual buffers with sizes $t=170,340$ and 510 cells respectively (which corresponds to $0.5,1.0$ and 1.5 burst lengths respectively), and the monitored data are shown as circles in Figure 3. The regression (1) using the SFF model (10) and its asymptotic model (6) generate the estimate "regression 1" and "regression 2", respectively. Figure 3 shows that the "regression 1" produces a much better estimate than "regression 2". For example at buffer size $t=4000$ cells, the CLR predicted by "regression 1" and "regression 2 " are $5.3 \times 10^{-8}$ and $3.2 \times 10^{-9}$ respectively, while the exact value by simulation is $1.2 \times 10^{-7}$. It is because the asymptotic model (6) assumed in "regression 2" only holds for large buffers. On the contrary, the "regression 1" using the SFF model can cover a wider range of buffer sizes and thus it produces a better estimate. Therefore, the results obtained from asymptotic model (6), such as the one used in [15], should be examined carefully.

\subsection{Reduced Capacity Example}

As mentioned in Section 3.3, reduced buffer approach may not work for monitoring self-similar traffic. In this case, we shall use the reduced capacity approach instead because reducing the service capacity always leads to a large increase in measured cell loss ratios. A trace driven simulation is used to study the performance of our regression method for VBR video sources. We consider the movie "Star Wars" [8] encoded in motion JPEG format with traffic characteristics shown in Table 2. The original sequence is 2 hours long and contains 171,000 frames. The frame rate is 24 frames/s and the cell size is assumed to be 48 bytes. Since we only have a limited number of real video traces, we use the method proposed in [8][10] to generate multiple video sources from a single trace. We consider the VBR trace as a circular list and then 
randomly pick a starting point in the list as the first frame of a video sequence. The starting point of each trace is chosen to be at least 4000 frames apart from each others.

Table 2: Characteristics of Video Sources

\begin{tabular}{lcccccc}
\hline \multirow{2}{*}{ Bit stream } & \multicolumn{4}{c}{ Bit stream statistics (bytes / frame) } & \multirow{2}{*}{\begin{tabular}{c} 
Sequence \\
length \\
\cline { 2 - 5 } (frames)
\end{tabular}} & $\begin{array}{c}\text { Hurst } \\
\text { parameter }\end{array}$ \\
\hline Star Wars & 78459 & 8622 & 27791 & 6254 & 171,000 & 0.78 \\
\hline
\end{tabular}

The Hurst parameter of this JPEG sequence is found to be 0.78 using the time-variance plot. This confirms that the source is self-similar as suggested in [8]. We have mentioned that buffering does not help much to reduce cell losses in video transmission. In addition, large buffer may introduce large queueing delay which is not desirable for real-time applications like video. We hence use a medium size buffer of 500 cells in the multiplexer.

Suppose we have 10 such video sources multiplexed into this buffer. The simple FIFO policy is adopted in this study, (i.e., cells arriving to a full buffer are simply dropped). Each source sends 24 frames per second and cell arrivals are equally spaced within each frame interval. Furthermore, we choose the arrival instants of frames from different sources to be uniformly spaced in the interframe interval. This can reduce the source-periodicity effect [10], which causes different sources to experience very different CLR in a FIFO queue. We run the simulation for 2500 seconds ( $=60,000$ frames) so that we can measure the CLR accurately up to the range of $10^{-6}$. For video transmission over ATM networks, the range of CLR requirement normally falls between $10^{-4}$ to $10^{-6}$.

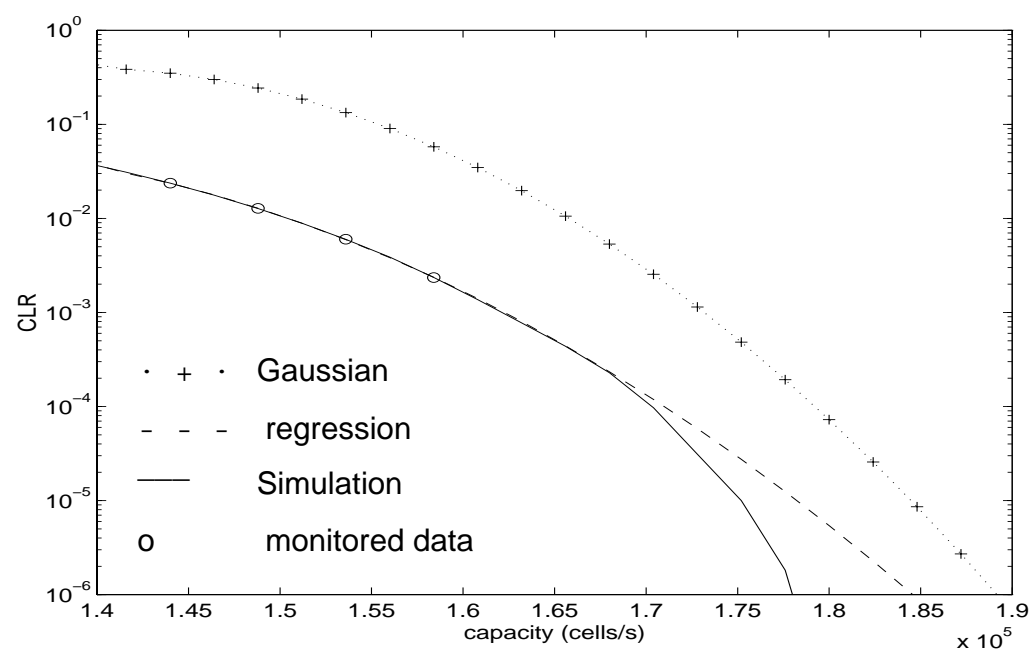

Figure 4. Comparison of regression approaches.

In [2], we find that the Gaussian approximation (8) is a good estimate of the required bandwidth for video sources. Accordingly, we take this as our model $m(t)$. It is shown in Figure 4 that the Gaussian approximation is a conservative estimate of the required bandwidth. We monitored the CLRs at four different capacities using virtual buffers and they are shown as small circle in Figure 4. Let $t$ be the capacity, $m(t)$ and $a(t)$ be the corresponding $\log (C L R)$ using Gaussian approximation and simulation, respectively.

It is clear from (8) that $\log (C L R)$ is a quadratic function of capacity with Gaussian approximation. If we apply (1) to perform the regression, the regressed estimate will give 
another quadratic function. Figure 4 shows that our regression overestimates the CLR compared to the exact curve but it is still much better than the original Gaussian approximation For example, at capacity $C=1.75 \times 10^{5}$ cells/s, CLR obtained by simulation is $1 \times 10^{-5}$, regressed value is $3 \times 10^{-5}$ and Gaussian prediction is $5 \times 10^{-4}$. However, the regressed estimate is getting worse for smaller CLR. It is because the curve of CLR vs capacity of the real trace drops at a much faster rate than that predicted by the quadratic function.

\subsection{CONCLUSION}

In this paper, we propose a new performance monitoring mechanism to monitor stringent CLR like $10^{-8}$ within a reasonable time window. We first monitor the CLR at several virtual buffers using the VOB method. These monitored data at virtual buffers are then combined with a model in a regression algorithm to estimate the actual CLR of the physical system. It shows that the simple linear regression formula can give accurate regressed estimate based on only 3 to 4 monitored performance.

\section{REFERENCES}

[1] D. Anick, D. Mitra, M.M. Sondhi, "Stochastic theory of a data handling system with multiple sources", Bell Syst. Tech. J., vol. 61, no. 8, 1992.

[2] H.W. Chu, D.H.K. Tsang and T. Yang, "Call admission control of teleconference VBR video traffic in ATM networks”, Proc. IEEE ICC’95, pp. 847-851, 1995.

[3] C. Courcoubetis, G. Fouskas, R. Weber, "An on-line estimation procedure for cell-loss probabilities in ATM links." IFIP Third Workshop on Performance Modelling and Evaluation of ATM Networks. U.K., (1995) p41/1-10.

[4] C. Courcoubetis, G. Kesidis, A. Ridder, J. Walrand and R. Weber, "Admission Control and Routing in ATM Networks using Inferences from Measured Buffer Occupancy", IEEE Trans. on Commun., vol. 43, no. 2/3/4, pp. 1778-1784, Feb-Apr. 1995.

[5] N.G. Duffield et. al., "Predicting quality of service for traffic with long-range fluctuations", Proc. IEEE ICC'95, pp. 473-477, 1995.

[6] N. G. Duffield and N. O'Connell, "Large deviations and overflow probabilities for the general single-server queue, with applications", Mathematical Proceedings of the Cambridge Philosophical Society, 1996.

[7] A. Elwalid, D. Heyman, T.V. Lakshman, D. Mitra and A. Weiss, "Fundamental Bounds and Approximations for ATM Multiplexers with Applications to Video Teleconferencing", IEEE J. Select. Areas Commun., vol. 13, no. 6, pp. 1004-1016, Aug. 1995.

[8] M. W. Garrett and W. Willinger, "Analysis, modeling and generation of self-similar VBR video traffic", Proc. SIGCOMM 94, pp. 269-280, 1994.

[9] R. Guerin, H. Ahmadi, and M. Naghshineh, "Effective capacity and its application to bandwidth allocation in high-speed networks," IEEE J. Select. Areas Commun., vol. 9, no. 7 pp. 968-981, 1991.

[10] D.P. Heyman, A. Tabatabai, and T.V. Lakshman, "Statistical analysis and simulation study of video teleconference traffic in ATM networks", IEEE Trans. on Circuits and Systems for Video Technology vol. 2, no. 1, pp. 49-59, 1992.

[11] A. Hiramatsu, "Training techniques for neural network applications in ATM", IEEE Commun. Mag., vol. 33, pp. 58-67, Oct. 1995.

[12] W. Leland, M. Taqqu, W. Willinger, and D. Wilson, "On the self-similar nature of ethernet traffic (extended version)", IEEE/ACM Trans. on Network., vol. 2, no. 1, pp. 1-15, Feb. 1994.

[13] J. W. Roberts, "COST 224: Performance evaluation and design of multiservice networks", 1991.

[14] H. Saito, "Bayesian Performance Estimation Driven by Performance Monitoring and Its Application", IEICE Trans. Commun., vol. E79-B, no. 1, pp. 1-7, Jan. 1996.

[15] H. Zhu and V. S. Frost, "In-Service Monitoring for Cell Loss Quality of Service Violations in ATM Networks", IEEE/ACM Trans. on Network., vol. 4, no. 2, April 1996, pp. 240-248. 